\title{
Philosophiques
}

\section{Motivations stratégiques et compétition fiscale}

\section{Louis-Philippe Hodgson}

Volume 43, numéro 1, printemps 2016

URI : https://id.erudit.org/iderudit/1036471ar

DOI : https://doi.org/10.7202/1036471ar

Aller au sommaire du numéro

Éditeur(s)

Société de philosophie du Québec

ISSN

0316-2923 (imprimé)

1492-1391 (numérique)

Découvrir la revue

Citer ce document

Hodgson, L.-P. (2016). Motivations stratégiques et compétition fiscale.

Philosophiques, 43(1), 119-125. https://doi.org/10.7202/1036471ar d'utilisation que vous pouvez consulter en ligne.

https://apropos.erudit.org/fr/usagers/politique-dutilisation/ 


\title{
Motivations stratégiques et compétition fiscale
}

\author{
LOUIS-PHILIPPE HODGSON
}

Université York

Dans un monde où les entreprises peuvent s'établir où elles le veulent et où les États sont largement libres de fixer leur politique fiscale comme ils l'entendent, un problème de compétition évident se pose. Chaque État a intérêt, afin d'attirer les investissements étrangers, à ce que son taux d'imposition sur les entreprises soit plus bas que celui des autres. Mais si chacun tente d'adopter un taux d'imposition plus bas que celui de son voisin, il s'ensuivra inévitablement une chute dramatique des taux d'imposition de tous, et donc une diminution générale des revenus. Chaque État verra ainsi sa situation empirée par la compétition fiscale que semblait pourtant lui enjoindre son intérêt.

Le problème se pose avec acuité dans le monde actuel, avec toutes les conséquences que l'on sait pour la capacité des États - et surtout des États les plus démunis - d'adopter des programmes sociaux répondant aux besoins de leurs citoyens. Le livre Catching Capital de Peter Dietsch, qui en présente une étude à un niveau de détail qui n'avait à ma connaissance pas été approché jusqu'à présent dans la littérature philosophique sur la question, arrive donc fort à propos ${ }^{1}$. Dietsch travaille à partir d'ouvrages d'économie et de science politique, de textes de loi et de rapports gouvernementaux qui vont bien au-delà des frontières habituelles de la philosophie politique, et il nous en présente les faits saillants avec assurance et clarté. Les spécialistes de la philosophie politique qui veulent en apprendre davantage sur cet aspect trop oublié de la justice internationale lui seront assurément reconnaissants de pouvoir désormais le faire sans avoir à parcourir une littérature dont on soupçonne, vu le sujet, que sa principale qualité n'est pas d'être captivante.

Il y aurait évidemment beaucoup à dire sur les nombreuses facettes du livre, mais je veux me concentrer ici sur ce qui en constitue à mon sens le noyau philosophique, à savoir le principe que présente Dietsch au chapitre II pour établir les limites acceptables de la compétition fiscale entre États. Il va comme suit:

Any fiscal policy of a state is unjust and should be prohibited if it is both strategically motivated and has a negative impact on the aggregate fiscal selfdetermination of other states (p. 80).

1. Peter Dietsch, Catching Capital: The Ethics of Tax Competition, Oxford, Oxford University Press, 20I 5 . Les références entre parenthèses dans le texte se rapportent toutes à cet ouvrage. 
Chacune des deux parties de ce principe soulève des questions philosophiques difficiles. Pourquoi la justice de la politique fiscale d'un État dépendrait-elle essentiellement des motivations ou des intentions ayant mené à son adoption? Et pourquoi la justice d'une politique fiscale donnée dépendrait-elle de son effet sur l'autonomie fiscale des autres États considérée de manière agrégée? Les deux suggestions n'ont rien d'évident. Je vais surtout me concentrer ici sur la première, qui me semble toucher à des enjeux particulièrement complexes, mais je présenterai également quelques remarques sur le rôle de l'agrégation dans l'approche de Dietsch.

Pour que la politique fiscale d'un État constitue une injustice envers les citoyens d'un autre État, il est nécessaire, selon Dietsch, que son adoption ait été motivée par des considérations stratégiques. La suggestion soulève une question philosophique d'un intérêt indéniable: dans quelle mesure les intentions qui ont présidé à l'adoption d'une politique publique ont-elles à voir avec la justice de cette politique? Dietsch maintient que les deux idées sont étroitement liées, mais un certain scepticisme me semble de mise.

On s'interroge bien sûr fréquemment sur les motivations qui ont pu mener à l'adoption d'une certaine politique. Songez aux innombrables spéculations sur l'invasion de l'Iraq par les États-Unis en 2003. Le président George W. Bush était-il mû par l'appât de l'or noir, par des sentiments vindicatifs envers celui qui avait jadis nargué son père, ou par un désir sincère de protéger la population iraquienne contre un tyran sanguinaire? La portion du peuple américain qui soutenait Bush était-elle mue par un désir de vengeance teinté de confusion après les attentats du II septembre, ou par des sentiments plus louables? Ce sont là des questions d'une importance indéniable, mais il faut être précis quant à la nature des enjeux qu'elles soulèvent. La part des motifs vindicatifs dans le raisonnement du président et de ses supporteurs a beaucoup à nous dire sur l'évaluation morale qu'il faut faire de leur caractère. Mais la question de savoir si l'invasion de l'Iraq était injuste tient vraisemblablement à des facteurs tout autres - par exemple, aux raisons qu'il y avait de croire que Saddam Hussein posait véritablement un danger grave pour ses citoyens ou pour le reste du monde, que l'invasion pouvait être menée avec succès, et que le risque de guerre civile pouvait être esquivé. Ces considérations n'ont, comme telles, rien à voir avec les dispositions psychologiques de Bush et de ses supporteurs. Si Bush était mû par des considérations purement stratégiques ou vindicatives, on peut certes en conclure qu'il n'aurait jamais dû se voir confier quelque position d'autorité que ce soit, mais la justice de la politique qu'il a adoptée n'est pas nécessairement affectée; il est toujours possible, en politique sans doute plus qu'ailleurs, de poser la bonne action pour de mauvaises raisons ${ }^{2}$.

2. Je ne veux surtout pas suggérer que l'invasion de l'Iraq était justifiée - simplement que la question ne dépend pas des motivations de ceux qui l'ont ordonnée. L'idée que j'avance ici est évidemment très proche de la distinction qu'établit T. M. Scanlon dans Moral Dimen- 
Qu'en est-il du rôle des intentions dans l'évaluation de la politique fiscale d'un État? Il est tentant de penser que le même type de raisonnement s'applique. Si l'Irlande réduit son taux d'imposition sur les entreprises à Io \% pour attirer plus d'investissements étrangers, et donc pour se donner un avantage stratégique par rapport aux autres États, on peut sans doute en conclure que le caractère de ses dirigeants, voire celui de l'électeur irlandais moyen, laisse à désirer. Mais cette donnée est-elle pertinente pour évaluer si l'adoption d'un tel taux d'imposition constitue, du point de vue des autres pays affectés, une injustice?

Il faut noter que Dietsch répond par l'affirmative pour une raison bien précise, à savoir, parce qu'il adopte comme valeur fondamentale la souveraineté fiscale et qu'il interprète cette valeur comme traçant une ligne claire entre le cas d'un pays qui réduit son taux d'imposition pour des raisons stratégiques et celui d'un pays qui le fait par conviction idéologique - ce dernier cas étant seul un exercice fondamental de souveraineté fiscale. Ainsi, selon Dietsch, si les Anglais veulent adopter un taux d'imposition plus bas que celui de la Suède parce qu'ils préfèrent des programmes sociaux moins généreux, il faut respecter cette décision au nom de la souveraineté fiscale; mais si les Irlandais font de même pour des raisons stratégiques, leur action ne saurait se mériter la même protection (p. 95-96).

D'un point de vue strictement intuitif, la distinction a quelque chose de séduisant. Je doute cependant qu'elle puisse réellement justifier le rôle que jouent les intentions dans la théorie de Dietsch. En fait, je suis enclin à croire qu'une optique résolument différente sur la question est préférable. $\mathrm{Au}$ bout du compte, tout pays qui participe au système économique global doit, lorsqu'il prend des décisions de politique économique, tenir compte de leur effet potentiel sur les autres membres du système. Le raisonnement est simple: la participation à un marché interétatique peut amener des bénéfices considérables, mais pour que les intérêts de tous soient promus, il est essentiel que chacun accepte certaines restrictions, et donc certains coûts. L'enjeu fondamental est de savoir ce qui constitue une répartition équitable des coûts et bénéfices en question.

La thèse défendue par Dietsch implique que les coûts que doit accepter un État pour participer au système économique global varient selon les dis-

sions entre permissibilité (permissibility) et signification (meaning) (voir T. M. Scanlon, Moral Dimensions: Permissibility, Meaning, Blame, Cambridge, Harvard University Press, 2008). La permissibilité d'une action - en gros, dans notre contexte, la question de savoir si l'action est compatible avec la justice ou pas - dépend des raisons qui favorisent l'action, mais pas (ou du moins pas de façon simple) des raisons qui, de fait, motivent l'agent. Ces dernières sont toutefois déterminantes pour la signification de l'action, qui concerne les répercussions que le fait qu'un agent ait posé une action pour des raisons données a sur les relations qu'on peut vouloir entretenir avec lui. Ainsi, si Bush a véritablement ordonné l'invasion de l'Iraq pour des motifs bassement personnels, on est assurément justifié de vouloir éviter de passer du temps avec lui et de ne pas vouloir lui confier quelque responsabilité que ce soit. 
positions idéologiques de ses citoyens. Cela me semble difficile à croire. Pensez au comité de résolution des disputes fiscales que Dietsch aimerait voir mettre en place (p. I I I). Pourrait-il vraiment appliquer des règles dont le contenu dépend aussi directement des intentions des participants que le requiert le principe de Dietsch ? Imaginez un peu ce comité tenant aux Irlandais un discours comme celui-ci: "Peuple irlandais, vous voulez adopter un taux d'imposition de so\% sur les entreprises. Les débats qui ont eu lieu dans votre parlement et dans vos journaux montrent clairement que vous espérez ainsi obtenir un avantage compétitif par rapport aux autres pays de l'Union européenne. Peuple irlandais, ce sont là des motifs purement stratégiques! Nous ne pouvons pas autoriser une telle politique. Bien sûr, vous rétorquerez que l'Angleterre a déjà adopté un taux d'imposition de ı०\% et que nous n'avons rien dit. Mais, justement, leur motivation n'était pas stratégique! Comme vous le savez sans doute, le livre Capitalism and Freedom a récemment fait un carton chez vos voisins. La population, mue par une ferveur idéologique rare, a décidé de ramener les programmes sociaux à un strict minimum. Ce choix démocratique doit clairement être protégé en vertu de l'importance fondamentale de la souveraineté fiscale; il n'en va pas de même de vos sombres machinations. »

Même si on laisse de côté la question de savoir s'il est plausible, dans le cadre d'une théorie qui accorde une importance centrale à la notion de souveraineté, de donner à un comité international l'autorité d'évaluer ainsi les intentions d'un État, le raisonnement semble absurde 3 . Si l'effet d'un taux d'imposition de ro\% sur les autres pays est suffisamment sérieux, alors l'interdiction d'adopter un tel taux est simplement un des coûts que doit accepter un pays pour faire partie de l'économie commune dont tous bénéficient. Je vois mal comment on pourrait croire que ce coût doive s'appliquer aux Irlandais mais pas aux Anglais. Faire ainsi varier le coût d'admission à l'économie commune selon les penchants idéologiques des participants me semble profondément inéquitable, et je doute fortement que

3. Dietsch reconnaît que l'évaluation des intentions d'un pays pose problème. Il suggère sur ce point qu'il faut trouver des critères objectifs pouvant agir comme indicateurs indirects des intentions (la nature exacte de ces critères doit, selon lui, être déterminée par les avocats qui se spécialisent dans les questions fiscales internationales (p. IO9-IIO); la manœuvre laisse, il faut le dire, un certain goût d'inachevé). Par ailleurs, Dietsch semble penser que le rôle joué par les intentions dans les décisions d'une instance comme la Cour internationale de justice montre que celui qu'il veut leur donner dans l'évaluation des politiques fiscales n'a rien de troublant (p. I Io). Le parallèle est loin d'être évident. D'une part, la Cour internationale de justice s'intéresse aux intentions des individus, et non à celles des États - la seconde notion étant évidemment bien plus problématique que la première. De plus, la question qui se pose dans une cause criminelle est simplement celle de savoir si l'action peut être attribuée à l'accusé (c'est-à-dire si elle était, sous la description appropriée, intentionnelle). Que l'action ait été commise pour des raisons stratégiques ou idéologiques n'est d'aucune pertinence, contrairement à ce qui est le cas dans l'approche de Dietsch, qui donne en ce sens un rôle beaucoup plus central aux intentions. 
la proposition puisse être rachetée en invoquant une quelconque notion de souveraineté fiscale.

Le rôle que doivent jouer les intentions dans la théorie de Dietsch est d'autant plus surprenant que celui-ci admet explicitement que les intentions stratégiques sont parfaitement acceptables lorsqu'elles mènent à des résultats bénéfiques. Ainsi, si la Belgique décide d'investir dans des infrastructures de premier ordre dans le but d'attirer plus d'investissements étrangers, la politique doit être autorisée (p. 97). Dietsch en conclut qu'il est essentiel que notre principe de justice fiscale tienne compte à la fois des intentions qui mènent à l'adoption d'une politique et des effets de la politique sur les autres pays. J'avoue être tenté par une conclusion différente. Je suis enclin à penser que le contraste entre le cas de l'Irlande et celui de la Belgique montre surtout que les intentions qui mènent à l'adoption d'une politique n'ont pas de rôle essentiel à jouer du point de vue de la justice. L'enjeu réel concerne plutôt le type de compétition dans lequel s'inscrit la politique. Si la compétition entraîne des bénéfices - s'il s'agit d'une "course vers le sommet" (race to the top) - alors il n'y a pas de raison d'interdire la politique. Par contre, si la compétition a des effets néfastes - s'il s'agit d'une "course vers l'abîme» (race to the bottom) - alors des règles claires doivent être mises en place pour la rendre compatible avec les intérêts de tous, ou du moins pour en atténuer les effets négatifs.

Dietsch souligne à plusieurs reprises que la compétition fiscale entraîne les États dans une course vers l'abîme. Je conserve l'impression que c'est là où tout se joue - que le véritable problème tient à la structure d'interaction qui précipite une telle course, et que la solution tient simplement aux règles qui permettraient de l'éviter, et non aux motifs suivant lesquels les participants agissent à l'intérieur de la structure en question. Après tout, le problème est de nature essentiellement hobbésienne, et l'extraordinaire puissance de l'argument de Hobbes tient justement au fait qu'il ne dépend pas des intentions des participants: deux personnes qui se retrouvent dans un état de nature seront poussées par la structure de leur situation à s'entretuer, peu importe la pureté des intentions qu'elles avaient au début de leur interaction.

Dietsch semble vouloir insister sur le fait que certaines politiques fiscales qui ont des effets néfastes doivent néanmoins être permises parce que leur adoption reflète un exercice concerté de souveraineté fiscale. Mais je vois mal pourquoi la communauté internationale n'aurait pas le droit d'adopter des règles interdisant toute politique fiscale qui risque de contribuer sérieusement au problème de la compétition fiscale. Après tout, si les Anglais adoptent un taux de ı०\% pour des raisons purement idéologiques, les Irlandais devront alors baisser le leur à $9 \%$; la pureté idéologique n'y change rien.

Je serais d'autant moins troublé par l'adoption de telles règles que, contrairement à Dietsch, je ne suis pas convaincu qu'il faille y voir une 
entorse à la souveraineté fiscale. Serait-il vraiment incompatible avec l'idée de souveraineté fiscale - dans la mesure, du moins, où cette idée mérite notre adhésion - de dire à un pays que, s'il veut participer à l'économie commune, il doit accepter de jouer selon certaines règles qui protègent les intérêts de tous? C'est loin d'être évident ${ }^{4}$. De plus, il vaut la peine de noter que les choix qui sont essentiels à la souveraineté fiscale selon Dietsch celui de la part que doit occuper l'économie publique dans l'économie totale d'un pays, et celui du niveau de redistribution des richesses qui doit y avoir lieu (p. 35 et p. I70) — n'ont pas nécessairement à passer par l'impôt sur les entreprises. Dans la mesure où une harmonisation des taux d'imposition sur les entreprises aurait été effectuée, et où chaque pays serait ainsi capable d'aller chercher une part de revenu appropriée par ce mécanisme ${ }^{5}$, je ne vois pas ce qui empêcherait les variations idéologiques entre pays de trouver leur expression dans les différents taux d'impôt sur le revenu des particuliers adoptés par les différents pays. Il n'est donc pas clair que l'impôt sur les entreprises doive occuper une place aussi importante dans notre conception de la souveraineté fiscale que le suggère Dietsch.

En maintenant qu'il faut nous concentrer sur l'effet des politiques fiscales et non sur les motivations des pays qui les adoptent, je ne cherche surtout pas à pousser Dietsch dans la direction d'une position plus conséquentialiste. Au contraire, je veux souligner en terminant que la seconde partie du principe de Dietsch - celle qui concerne l'effet des politiques fiscales - semble poser problème justement parce qu'elle fait intervenir une idée proprement conséquentialiste que nous devrions rejeter, à savoir que la souveraineté fiscale des pays doit être considérée de manière agrégée.

Cet aspect de l'approche de Dietsch est particulièrement surprenant, il faut le dire, puisque la souveraineté est généralement comprise comme une valeur qui demande à être respectée plutôt que promue, et donc comme une valeur qui, par nature, résiste à la logique agrégative. Il faut ajouter à cela que les raisons qui poussent Dietsch vers une approche conséquentialiste sont loin d'être claires. Le passage où il les discute (p. 99-I00) est malheureusement expédié de manière trop sommaire pour répondre aux questions

4. Je ne suis pas tout à fait certain d'avoir bien saisi la position de Dietsch sur ce point. Par moments, il semble rejeter l'idée d'une harmonisation des taux d'imposition sur les entreprises au niveau international, puisqu'une telle approche constituerait une violation de l'autonomie fiscale des États (voir p. 62). À d'autres moments, il semble dire non seulement qu'une telle harmonisation pourrait être acceptable, mais encore qu'il faudrait y voir, non pas une restriction, mais bien une condition de la souveraineté fiscale proprement comprise (voir p. I78 sq.).

5. La précision est importante. Dietsch souligne avec justesse que les pays pauvres ne sont généralement pas capables de compenser les pertes de revenus en impôt sur les entreprises par une augmentation de l'impôt sur le revenu des particuliers (voir notamment p. 48 sq.). Ma suggestion est simplement que les aspects de la souveraineté fiscale que veut protéger Dietsch peuvent être réalisés par le biais de l'impôt sur les particuliers, mais seulement dans la mesure où des règles appropriées ont d'abord résolu le problème de la compétition fiscale. 
que se posera le lecteur qui n'est pas déjà acquis à la cause conséquentialiste. Une remarque qu'y fait Dietsch pourrait même alimenter ces questions. Dietsch souligne (p. I०০) que son critère doit s'appliquer de façon rétrospective: il propose une procédure par laquelle un pays peut se plaindre des effets que la politique fiscale d'un autre pays a déjà eu, et non de ses effets anticipés (lesquels seraient, selon Dietsch, trop difficiles à évaluer). La question semble alors particulièrement pressante: si l'on admet une telle façon de procéder, pourquoi exiger que la politique ait eu un effet négatif, non pas seulement sur la souveraineté fiscale du pays qui porte plainte, mais sur la souveraineté fiscale de tous les pays considérée de manière agrégée?

Il est bien sûr probable qu'une politique fiscale qui nuit à un pays donné nuira également à d'autres. Mais considérons le type de cas qui pose habituellement problème pour les approches conséquentialistes: un scénario où la souveraineté fiscale d'un seul pays serait fortement compromise par la politique fiscale de l'Irlande (par exemple), mais où celle de nombreux autres pays serait très légèrement promue, avec pour résultat que l'effet sur la souveraineté fiscale de tous les pays, considérée de manière agrégée, serait positif. Est-il vraiment plausible de soutenir que le pays dont la souveraineté fiscale a été compromise n'aurait alors pas d'objection valable contre la politique en question?

Il aurait été intéressant que Dietsch discute en détail des différentes options qui s'offrent à nous pour évaluer une telle situation. Pourquoi ne pas dire qu'un pays peut déposer une plainte simplement en vertu de l'effet qu'une politique fiscale a sur ses intérêts fiscaux? Si une telle approche risque de créer trop de complications, pourquoi ne pas se concentrer simplement sur l'effet de la politique sur les pays les moins bien nantis? De prime abord, ces approches me semblent toutes deux préférables au conséquentialisme de Dietsch, puisqu'elles nous permettent non seulement de prendre au sérieux l'intérêt fiscal de chaque pays, mais également de reconnaître qu'une population dont la souveraineté fiscale a été sérieusement compromise n'est aucunement compensée lorsque la souveraineté fiscale de la population voisine est accrue par une magnitude supérieure.

En terminant, je veux souligner encore une fois que les remarques que j'ai présentées ici sont évidemment beaucoup trop pointues pour rendre compte de la diversité des enjeux que Dietsch aborde dans Catching Capital et de la richesse du traitement qu'il en donne. Cet ouvrage est manifestement le fruit d'une réflexion assidue; même ceux qui, comme moi, ont des réserves sur les détails du cadre normatif dans lequel Dietsch travaille lui sauront gré d'avoir exploré avec autant de détermination des questions que les philosophes politiques avaient jusqu'à présent trop souvent ignorées. 